\title{
The Pseudorabies Virus Encoded Serine/Threonine Kinase Us3 and Viral Egress
}

\author{
L.M. Olsen*, L.W. Enquist* and M.E. Bisher* \\ *Department of Molecular Biology, Princeton University, Princeton, NJ 08544
}

The herpesvirus family is defined by a common infection pattern and viral particle architecture. The virion is composed of three distinct structural entities. The exposed outer surface of the virion consists of a lipid bilayer derived from cellular membranes and contains a dense collection of viral transmembrane proteins. This surrounds an icosahedral capsid that carries the viral genome. Between the envelope and capsid lies a layer of virally encoded proteins collectively know as the tegument. The trafficking of the assembling particle through the cell during its acquisition of virion components defines the egress pathway. DNA filled capsids are assembled in the nucleus then bud into the inner nuclear envelope. During this primary envelopment step, virions acquire a layer of primary tegument and form a 'primary enveloped' intermediate in the perinuclear space. Via fusion with the outer nuclear envelope this particle de-envelops and is released into the cytoplasm. The acquisition of cytoplasmic tegument proteins occurs through a web of protein-protein interactions. Finally, the virion undergoes secondary envelopment upon budding into a trans-golgi like compartment containing viral membrane glycoproteins. Fusion of this compartment with the plasma membrane releases infectious particles into the extracellular environment.

Alpha-herpesviruses, such as pseudorabies virus, encode a unique protein important in the primary envelopment steps of egress. This serine/threonine kinase, Us3, is non-essential in tissue culture but has a cell type dependent replication defect as well as an extracellular particle 'release' defect. Interestingly, Us 3 is acquired in the nucleus and is one of the only tegument components found in both the primary and secondary tegument [1]. Us3 also orchestrates the localization of several viral proteins to the inner nuclear envelope and in PRV infected explants of nasal epithelia, Us3 mutants are impaired in the de-envelopment of primary enveloped particles in the perinuclear space [2,3]. In this study we examined infected cells at late times during infection to identify the possible roles of Us3 in virion assembly and egress. PK15 cells were infected for 16 hours then scraped, pelleted, and processed for conventional transmission electron microscopy.

Our results indicate that the peri-nuclear, de-envelopment delay previously seen in primary explant tissue also occurs in the porcine kidney tissue culture (PK15) cell line. Providing an easier culture system in which to investigate this phenotype. In addition, the secondary envelopment process in Us3null infections also appears compromised. In contrast to wildtype infection in which tegumented cytoplasmic particles readily line cytoplasmic vesicles and undergo secondary envelopment, Us3null particles appear disordered within the cytoplasm. These results indicate Us3 may not only facilitate de-envelopment in the primary stage of egress but also play a role in cytoplasmic tegument acquisition or targeting necessary for efficient secondary envelopment

\section{References:}

[1] H. Granzow et al. J. Virol. 78 (2004) 1314-1323

[2] B. Klupp et al. J. Gen Virol. 82 (2001) 2363-2371

[3] F. Wagenaar et al. J. Gen. Virol. 76 (1995) 1851-1859 

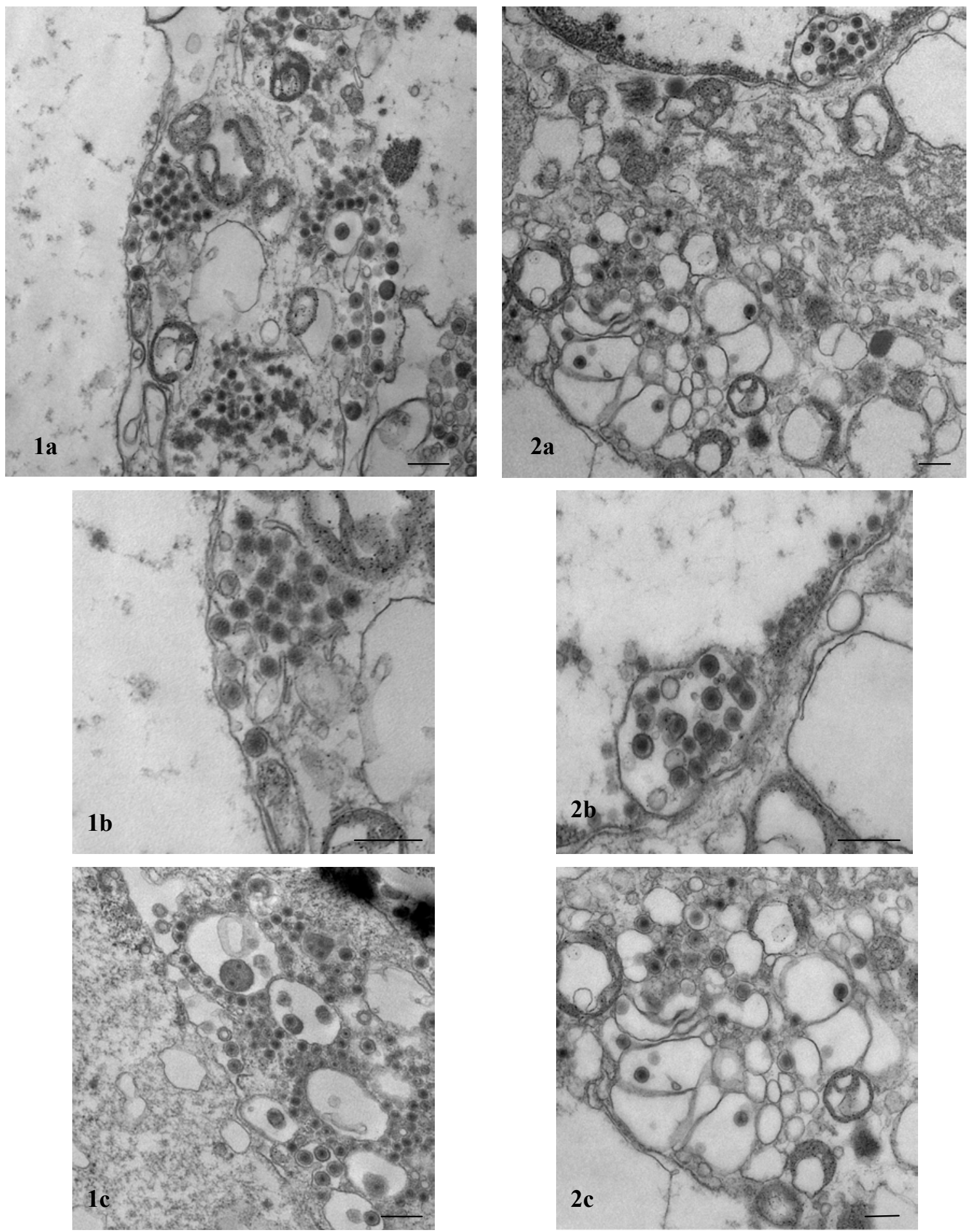

Figure 1: PK15 cells infected with wildtype (Becker) PRV. Whole cell image (a), Primary Envelopment (b) and Secondary Envelopment (c). Bar $=500 \mathrm{~nm}$

Figure 2: PK15 cells infected with US3null mutant. Whole cell image (a), Primary Envelopment (b) and Secondary Envelopment (c). Bar $=500 \mathrm{~nm}$ 